\title{
Tight Optimistic Estimates for Fast Subgroup Discovery
}

\author{
Henrik Grosskreutz, Stefan Rüping, and Stefan Wrobel \\ Fraunhofer IAIS, Schloss Birlinghoven, 53754 St. Augustin, Germany \\ \{henrik.grosskreutz, stefan.rueping, stefan.wrobel\}@iais.fraunhofer.de
}

\begin{abstract}
Subgroup discovery is the task of finding subgroups of a population which exhibit both distributional unusualness and high generality. Due to the non monotonicity of the corresponding evaluation functions, standard pruning techniques cannot be used for subgroup discovery, requiring the use of optimistic estimate techniques instead. So far, however, optimistic estimate pruning has only been considered for the extremely simple case of a binary target attribute and up to now no attempt was made to move beyond suboptimal heuristic optimistic estimates. In this paper, we show that optimistic estimate pruning can be developed into a sound and highly effective pruning approach for subgroup discovery. Based on a precise definition of optimality we show that previous estimates have been tight only in special cases. Thereafter, we present tight optimistic estimates for the most popular binary and multi-class quality functions, and present a family of increasingly efficient approximations to these optimal functions. As we show in empirical experiments, the use of our newly proposed optimistic estimates can lead to a speed up of an order of magnitude compared to previous approaches.
\end{abstract}

\section{Introduction}

Subgroup discovery Klö96. Wro97 is the task of finding subgroups of a population with high generality and distributional unusualness. It is a general approach that has shown to be useful in a variety of application scenarios, like medical consultation systems ABP06, spatial analysis KM02, marketing campaign planning LCGF04, and also in contrast set mining tasks KLGK07.

Unfortunately, if applied to real-world problems, subgroup discovery quickly results in excessive computation, due to its exponential dependency on the number of attributes. Different approaches have proposed to cope with that problem: While sampling based approaches SW00] relax the task by allowing a certain degree of departure from the optimal solution and a (controllable) error probability, other approaches make use of sophisticated data structures [AP06] or heuristics Klö02, LKFT04.

Another approach, proposed by Wrobel in [Wro97, is to prune the search space using so-called optimistic estimates. An optimistic estimate is a function that, given a subgroup $s$, provides a bound for the quality of every subgroup $s^{\prime}$ that is a refinement of $s$. Surprisingly, the use of optimistic estimates for fast

W. Daelemans et al. (Eds.): ECML PKDD 2008, Part I, LNAI 5211, pp. 440-456, 2008.

(C) Springer-Verlag Berlin Heidelberg 2008 
subgroup discovery has not yet been developed into a mature technology: until recently optimistic estimates have only been considered for the extremely simple case of a binary target attribute, and even in this case no attempt was made to move beyond suboptimal optimistic estimates.

In this paper, we investigate the question whether the optimistic estimates considered so far provide bounds that are, in some sense, optimal. To this end we provide a formal definition of tight optimistic estimates, that is optimistic estimates that are as conservative as possible wrt. the information at hand, namely the size of a subgroup and its distribution over different classes. Using this definition, we show that the optimistic estimate proposed in Wro97 is not tight. Thereafter, we present new tight optimistic estimates for some of the most common quality functions. We also present a family of increasingly efficient approximations to these optimal functions. While these optimistic estimates are not tight, they have the advantage that they are simpler to calculate.

Summarizing, the main contributions of this paper are thus (i) the formal definition of tightness, (ii) new, tight optimistic estimates for some of the most common quality functions, and (iii) a family of increasingly efficient approximations to these optimal functions. In an experimental section, we show that our results are not only interesting from a theoretical point of view, but also have a significant impact on the performance of subgroup discovery algorithms.

The paper is organized as follows: In section 2, we define the basics of the subgroup discovery task. In section 3 we provide our definition of tight optimistic estimates; thereafter, we present and prove new (tight) optimistic estimates. Section 4 contains the experiments, while section 5 concludes.

\section{Preliminaries}

In this section, we will introduce our terminology, formally define the problem of subgroup discovery, and motivate the concept of optimistic estimates 1

\subsection{The Task of Subgroup Discovery}

Let $D B=\left\{R_{1}, \ldots, R_{N}\right\}$ be a database or dataset, consisting of $N$ rows, each built up from of $l+1$ values. We distinguish one attribute $c$, called the class attribute with domain $D(c)=\left\{c_{1}, \ldots c_{m}\right\}$, from the $l$ ordinary attributes $\left\{a_{1}, a_{2}, \ldots a_{l}\right\}$ with domains $D\left(a_{i}\right)=\left\{v_{i, 1}, \ldots, v_{i, m_{i}}\right\}$. Every database row $R_{j}$ is an $n$-tuple $\left(v_{j, 1}, \ldots, v_{j, l}, c_{j}\right)$, and we call $c_{j}$ its class.

A subgroup description $s d$ is a set of terms $\left\{t_{1}, \ldots, t_{k}\right\}$ where every term $t_{i}$ is a constraint on an attribute, i.e. $t_{i}$ has the form $\left(a_{i}=v_{i}\right), v_{i} \in D\left(a_{i}\right)$. The length of the subgroup description is the number of terms it is built of. We call a subgroup description $s d^{\prime}=\left\{t_{1}^{\prime}, \ldots, t_{k^{\prime}}^{\prime}\right\}$ a refinement of a subgroup description $s d=\left\{t_{1}, \ldots, t_{k}\right\}$, denoted by $s d^{\prime} \succ s d$, if $\left\{t_{1}, \ldots, t_{k}\right\}$ is a subset of $\left\{t_{1}^{\prime}, \ldots, t_{k^{\prime}}^{\prime}\right\}$.

\footnotetext{
${ }^{1}$ In the following presentation, we assume that a dataset is provided as a single table. However, the concept of (tight) optimistic estimates also applies to the multirelational setting involving joins over relations as considered in Wro97.
} 
Given a database $D B$ and a subgroup description $s d$, the subgroup extension of $s d$ on $D B$ is the set of rows $R_{j} \in D B$ that satisfy all terms $t_{i} \in s d$. Please note that if $s d^{\prime}$ is a refinement of $s d$, i.e. $s d^{\prime} \succ s d$, then for every database $D B$ the subgroup extension for $D B$ and $s d^{\prime}$ is a subset of the subgroup extension for $D B$ and $s d$.

Given a set of rows $R=\left\{R_{1}, \ldots R_{n}\right\}$ (a database or subgroup extension), we call $n$ its size and $\boldsymbol{p}=\left(p_{1}, \ldots, p_{m}\right)$, where $p_{i}$ is the fraction of the rows of $R$ of class $i$, its class distribution. Formally, $\boldsymbol{p}$ is defined as follows:

$$
p_{i}:=1 / n \times|\{r \mid r \in R \wedge \operatorname{class}(r)=i\}| .
$$

Figure 1 shows an example with hypothetical data, inspired from a medical domain. The rows represent medical prescriptions made by doctors. As class attribute, we consider the cost of the prescription. Beside this special attribute, the prescriptions contain the doctor's specialty and the information whether the doctor's practice is in an urban or a rural environment. In this example, $\{$ Specialty $=$ Surgery $\}$ and $\{$ Specialty $=$

\begin{tabular}{lll} 
Cost & Specialty & Region \\
\hline High & Surgery & Urban \\
Medium & Internal Med Urban \\
Medium & Psychiatry & Urban \\
Medium & Internal Med & Rural \\
Low & Surgery & Rural \\
Low & Surgery & Rural
\end{tabular}

Fig. 1. Prescription example Surgery, Region $=$ Urban $\}$ are two subgroup descriptions, and the corresponding subgroups consist of the rows that fulfill these conditions. The size of the subgroup extension of $\{$ Specialty $=$ Surgery $\}$ is 3 and its probability distribution is $p_{\text {high }}=0.33, p_{\text {medium }}=0, p_{\text {low }}=0.66$.

A quality function $q$ is a mapping from $D B \times s d$ to the reals. Intuitively, a quality function expresses how "interesting" a subgroup is. Almost all quality functions considered in the literature only depend on some parameters of the subgroup and the database, in particular on the size $n$ of the subgroup, the size $N$ of the database, the class distribution $\boldsymbol{p}$ of the subgroup, and the class distribution $\boldsymbol{p}_{\mathbf{0}}$ of the database. Table 1 summarizes some of the most prominent quality functions [Klö96, SW00]: the Piatetsky-Shapiro quality function dealing with the two-class case, and the Split, Gini and Person $\chi^{2}$ quality functions for n-ary class attributes 2

The problem of subgroup discovery is defined as follows: Given a database $D B$, a quality function $q$, and a number $k$, determine the $k$ subgroup descriptions with maximum quality. Or, put more formally: return a set of $k$ subgroup descriptions $G$ such that

$\forall$ subgroup descriptions $s d: s d \notin G \Rightarrow q(D B, s d) \leq q *$,

where $q *=\min _{s d \in G} q(D B, s d)$.

${ }^{2}$ The notation and definitions used in other papers like KLJ03, Wro97. sometimes slightly differ from ours, but are (factor-) equivalent. For example, the Gini-Quality is often expressed using the generality (i.e. $n / N$ ) of the subgroup. Other authors use a notation like $p($ class $\mid s)$ to denote the probability of a class in a subgroup. 
Table 1. Common quality functions for subgroups

\begin{tabular}{lcl} 
NAme & Type Definition \\
\hline PiATETSKY-ShapiRo & 2 & $n\left(p-p_{0}\right)$ \\
SPlit & $\mathrm{N}$ & $n \sum_{i}\left(p_{i}-p_{0_{i}}\right)^{2}$ \\
GINi & $\mathrm{N}$ & $\frac{n}{N-n} \sum_{i}\left(p_{i}-p_{0_{i}}\right)^{2}$ \\
PEARSON'S $\chi^{2}$ & $\mathrm{~N}$ & $n \sum_{i} \frac{\left(p_{i}-p_{0_{i}}\right)^{2}}{p_{0 i}}$
\end{tabular}

\subsection{Optimistic Estimates and Their Use in Subgroup Discovery}

Before we present the definition of optimistic estimates, we would like to motivate this concept by taking a look at possible algorithmic approaches to subgroup discovery. Given that the space of candidate subgroup descriptions can be considered as a tree with subgroup descriptions of length 1 at the first level, subgroup descriptions of length 2 at the next level and so on, one obvious approach to subgroup discovery is to perform some kind of search.

Of course, in this approach the size of the search space is exponential in the number of attributes and hence it is desirable to use some kind of pruning strategy. Unfortunately, unlike related tasks like frequent item mining where state-of-the art algorithms like FpGrowth [HPYM04 exploit the property of monotonicity, in subgroup discovery this property does not hold: Even if the subgroup description $a_{1}=x$ does not have a sufficient quality, it is still necessary to consider its refinements. In fact, even if neither $a_{1}=x$ nor $a_{2}=y$ are interesting subgroups, $\left(a_{1}=x, a_{2}=y\right)$ might very well be interesting.

However, if we have already found $k$ subgroups and we knew that all refinements $s^{\prime}$ of a subgroup $s$ had a quality that is worse than that of all $k$ subgroups found so far, we could safely prune that branch. What is needed to do so is an optimistic estimate for the refinements $s^{\prime}$ of $s$ Wro97]:

Definition 1. An optimistic estimate oe(s) for a given quality function $q$ is a function that satisfies the following: $\forall$ subgroups $s, s^{\prime} . s^{\prime} \succ s \Longrightarrow$ oe $(s) \geq q\left(s^{\prime}\right)$.

\section{Tight Optimistic Estimates}

In this section, we will present our definition of tight optimistic estimates. Thereafter, we will present new optimistic estimates for all quality functions in Table 1

\subsection{A Definition of Tightness with Respect to Probability and Size}

The quality functions from Table 1 are all defined in terms of a few characteristics of the subgroup and the dataset, namely

- the distributions over the classes in the subgroup, denoted by $\boldsymbol{p}$;

- the size of the subgroup, denoted by $n$;

- the distributions over the classes in the dataset, denoted by $\boldsymbol{p}_{\mathbf{0}}$; and

- the size of the dataset, denoted by $N$. 
We will call such quality functions "probability/size quality functions" or "p/n quality functions". Formally, a $\mathrm{p} / \mathrm{n}$ quality function is a function $q\left(\boldsymbol{p}, n, \boldsymbol{p}_{\mathbf{0}}, N\right)$ from $[0,1]^{c} \times N \times[0,1]^{c} \times N$ to the reals (here, $c$ is the number of classes). We call $\boldsymbol{p}$ and $n$ the parameters of the subgroup and $\boldsymbol{p}_{\mathbf{0}}$ and $N$ the parameters of the overall population. Similarly, we will call optimistic estimates that only make use of these parameters "p/n optimistic estimates". Formally, a p/n optimistic estimate is a function from $[0,1]^{c} \times N \times[0,1]^{c} \times N$ to the reals such that

$$
\forall \text { subgroups } s, s^{\prime} . s^{\prime} \succ s \Longrightarrow o e\left(\boldsymbol{p}(s), n(s), \boldsymbol{p}_{\mathbf{0}}, N\right) \geq q\left(\boldsymbol{p}\left(s^{\prime}\right), n\left(s^{\prime}\right), \boldsymbol{p}_{\mathbf{0}}, N\right) \text {. }
$$

Here, $\boldsymbol{p}(s)$ and $n(s)$ denote the class distribution and the size for subgroup $s$. In general, there are infinitely many optimistic estimates. We are interested in optimistic estimates that are as conservative as possible in the following sense:

Definition 2. Given a quality function $q$ and two optimistic estimates oe 1 and oe $_{2}$, we call oe is $_{1}$ more conservative than oe 2 if $\forall N, \boldsymbol{p}_{\mathbf{0}}, n, \boldsymbol{p} . \mathrm{oe}_{\mathbf{1}}\left(\boldsymbol{p}, n, \boldsymbol{p}_{\mathbf{0}}, N\right) \leq$ $o e_{2}\left(\boldsymbol{p}, n, \boldsymbol{p}_{\mathbf{0}}, N\right)$.

The more conservative an optimistic estimate, the larger part of the search space can potentially be pruned: if we have already found $k$ subgroups with a minimum quality $\min Q$, then we can prune the branch of subgroups below $s$ if and only if $o e(s)<\min Q$. We will now formally define the notion of tight optimistic estimates, i.e. optimistic estimates that are as conservative as possible:

Definition 3. An optimistic estimate oe for a quality function $q$ is tight if for any population $D B$ and any subgroup description sd there is a subset $s^{\prime}$ of the extension of sd on $D B$ such that the quality of $s^{\prime}$ is equal to the optimistic estimate for sd on DB. Formally: the optimistic estimate oe is tight iff

$$
\forall D B, s d . \exists n^{\prime}, \boldsymbol{p}^{\prime} .\left[n^{\prime} \leq n \wedge n^{\prime} \boldsymbol{p}^{\prime} \preceq n \boldsymbol{p} \wedge o e\left(\boldsymbol{p}, n, \boldsymbol{p}_{\mathbf{0}}, N\right)=q\left(\boldsymbol{p}^{\prime}, n^{\prime}, \boldsymbol{p}_{\mathbf{0}}, N\right)\right] .
$$

Here, $\boldsymbol{p}_{\mathbf{0}}$ and $\boldsymbol{p}$ denote the probability distribution in $D B$, respectively in the extension of sd on $D B$, while $N$ and $n$ denote the size of $D B$ respectively of the subgroup extension (actually, $\boldsymbol{p}, n, \boldsymbol{p}_{\mathbf{0}}$ and $N$ are functions of $D B$ and $s$ ). $n^{\prime} \boldsymbol{p}^{\prime} \preceq n \boldsymbol{p}$ means that for all $i, n^{\prime} p_{i}^{\prime} \leq n p_{i}$, i.e. the number of rows of class $i$ in the subset of sd must be no larger than the number of rows of class $i$ in sd.

Please note that the above definition does only require that there is a subset of the extension of $s d$ on $D B$ with quality $o e\left(\boldsymbol{p}, n, \boldsymbol{p}_{\mathbf{0}}, N\right)$ - it does not require that there actually is a subgroup description with that quality. That is, the definition considers every subset of rows that is consistent with the restrictions provided by the parameters $\boldsymbol{p}$ and $n$. It is obvious that if an optimistic estimate for $q$ is tight, then there is no optimistic estimate for $q$ that is more conservative.

\subsection{A Tight Estimate for Piatetsky-Shapiro}

We will now apply our definition of tightness to the optimistic estimate published in Wro97 for the Piatetsky-Shapiro function, and show that it is not tight. Thereafter, we will present a tight optimistic estimate for that quality function. First, we remark that in this two-class case we use $p$ resp. $p_{0}$ to refer to the first component of $\boldsymbol{p}$ resp. $\boldsymbol{p}_{\mathbf{0}}$. 
Lemma 1. The optimistic estimate $n\left(1-p_{0}\right)$ presented in Wro97 for the Piatetsky-Shapiro function $n\left(p-p_{0}\right)$ is not tight. The optimistic estimate oe $e_{p s}^{*}:=$ $n p\left(1-p_{0}\right)$ is tight.

Proof. We first show that $o e_{p s}^{*}$ is tight. Suppose we are given a database $D B$ and an arbitrary subgroup extension $s$ with probability $p$ and size $n$. The case $p=0$ is trivial, so let $p>0$; The subgroup extension $s$ contains $n p$ rows of the first class. These rows are a subset of $s$ with size $n p$ and a class distribution of $p=1$; thus, this subset has quality $n p\left(1-p_{0}\right)$. This construction did not make any assumptions on $D B$ or $s$, thus there is always a subset $s^{\prime}$ with $q\left(s^{\prime}\right)=o e_{p s}^{*}(s)$, and $o e_{p s}^{*}$ is tight.

Finally, to see that $n\left(1-p_{0}\right)$ is not tight it is sufficient to note that $n p\left(1-p_{0}\right)<$ $n\left(1-p_{0}\right)$ for some $n>0, p_{0}<1$ and $p<1$.

\subsection{Tight Estimates for the Multi-class Quality Functions Split, Gini and Pearson's $\chi^{2}$}

Next, we turn to the multi-class quality functions. First, please note that all multi-class quality functions $q\left(\boldsymbol{p}, n, \boldsymbol{p}_{\mathbf{0}}, N\right)$ considered can be reformulated as functions $q\left(\boldsymbol{m}, \boldsymbol{p}_{\mathbf{0}}, N\right)$, where $\boldsymbol{m}=\left(m_{1}, \ldots, m_{c}\right)$ is a vector whose components are the numbers of rows of the different classes $1, \ldots, c$. The $m_{i}$ 's can be obtained from $\boldsymbol{p}, n$ by taking the scalar product $n \cdot\left(p_{1}, \ldots, p_{c}\right)^{T}$, while $\boldsymbol{p}$ and $n$ can be obtained from the $m_{i}$ 's by calculating $n=\sum_{j} m_{j}$ and $\boldsymbol{p}=\frac{1}{n} \cdot \boldsymbol{m}$. Using this new representation, we can now present a scheme of tight optimistic estimates for the multi-class quality functions in Table 1 :

Lemma 2. The following is a tight optimistic estimate for every multi-class quality function $q$ in Table 1 (in fact, for any quality function that is convex in $\boldsymbol{p}$ resp. $\boldsymbol{m})$ :

$$
o e_{q}^{*}\left(p_{1}, \ldots, p_{c}, n, \boldsymbol{p}_{\mathbf{0}}, N\right):=\max _{m_{1}^{\prime}, \ldots, m_{c}^{\prime} \mid m_{i}^{\prime} \in\left\{0, n p_{i}\right\}}\left\{q\left(\left(m_{1}^{\prime}, \ldots, m_{c}^{\prime}\right)^{T}, \boldsymbol{p}_{\mathbf{0}}, N\right)\right\}
$$

The above is thus the maximum over the $2^{c}$ possible combinations of values for $m_{1}^{\prime}, \ldots, m_{c}^{\prime}$, resulting from the constraint that every $m_{i}^{\prime}$ can either take the value 0 or $n p_{i}$. Please note that although not specified in Equation 1, the case $m_{i}^{\prime}=0$ for every $i$ needs not be considered.

Proof. The proof that $o e_{q}^{*}$ is an optimistic estimate is based on the fact that all the multi-class quality functions considered in Table 1 are convex in $\boldsymbol{p}$ resp. $\boldsymbol{m}$.

First, we use the fact that by definition a tight optimistic estimate for the refinements of a subgroup $s$ is the maximum over the quality of every possible subset of subgroup $s$. The subgroup $s$ has $n p_{1}$ rows of class $1, n p_{2}$ rows of class 2 , and so on. Thus, every refinement $s^{\prime}$ of $s$ consists of at most $n p_{i}$ rows of class $i$. Hence, a tight optimistic estimate for a quality function $q$ can be calculated as follows:

$$
\max _{m_{1}^{\prime}, \ldots, m_{c}^{\prime} \mid \forall i . m_{i}^{\prime} \in N_{+} \wedge 0 \leq m_{i}^{\prime} \leq n p_{i}}\left\{q\left(\left(m_{1}^{\prime}, \ldots, m_{c}^{\prime}\right)^{T}, \boldsymbol{p}_{\mathbf{0}}, N\right)\right\}
$$


Please note that unlike in Equation 1 the above considers the maximum over every subset of rows of the subgroup $s$. The above expression is not only an optimistic estimate, but it is tight, which follows directly from our definition of tightness from Section 3.1 .

It remains to show that Equations 1 and 2 are equivalent. This can be shown using the fact that every quality function in Table 1 is convex in its parameters $\boldsymbol{m}$. In fact, for every $c$-dimensional convex function $f$ the maximum over a polyhedron $P=\left[0, m_{1}\right] \times\left[0, m_{2}\right] \times \ldots \times\left[0, m_{c}\right]$ is an extreme point of $P$, also called a vertex Bre96, BV04. Thus, the maximum over $P$ can be calculated by taking the maximum of the values at every extreme point. The proof is completed by the fact that every quality function in Table 1 is convex, as shown in Appendix A. Please note that although in the Appendix we consider the extension of the quality functions to the real numbers, the extreme points are nevertheless tuples of positive natural numbers.

Let us now consider the computational complexity for the calculation of the tight optimistic estimate. $o e_{q}^{*}$ involves taking the maximum of $2^{c}$ values of $q$, where $c$ is the number of classes. All multi-class quality functions we considered can be reformulated to the form $q=\phi_{1}+\ldots+\phi_{c}$ (for the Split quality function, the expressions $\phi_{i}$ summed up are $n\left(p_{i}-p_{0 i}\right)^{2}$, for Gini they are $\frac{n}{N-n}\left(p_{i}-p_{0_{i}}\right)^{2}$ and for Pearson's they are $\left.\frac{n}{p_{0}}\left(p_{i}-p_{0_{i}}\right)^{2}\right)$. The calculation of such an expression $\phi_{i}$ involves only a constant number of subtractions and multiplications, thus the tight optimistic estimates $o e_{q}^{*}$ have a computational complexity of $O\left(c 2^{c}\right)$ primitive (add/multiply) operations. Please note that the computational complexity of $o e_{q}^{*}$ does not depend on the size of dataset, but only on the parameters $\boldsymbol{p}, n, \boldsymbol{p}_{\mathbf{0}}$ and $N$, which have to be calculated anyway to compute the quality of the subgroup.

\subsection{A Family of Increasingly Conservative Optimistic Estimates}

For large numbers of classes, $c$, the computational complexity, $O\left(c 2^{c}\right)$, of the tight optimistic estimate $o e_{q}^{*}$ can become problematic, as will be confirmed by the experiments in Section 4 .

In this section, we present a scheme of optimistic estimates that are not tight, but faster to calculate. The estimates are increasingly conservative and at the same time increasingly complex to calculate. The idea is not to consider all $2^{c}$ combinations as done in $o e_{q}^{*}$, but instead to consider only combinations for $d$ classes at a time. That is, we consider $2^{d}$ different combinations for the $d$ selected classes; for the other classes, we only consider the two extreme cases where either every $m_{i}^{\prime}=m_{i}$ or every $m_{i}^{\prime}=0$ (for classes $i$ not within the $d$ classes). Finally, the sum over all these maximums is calculated and used as an estimate 3

Here is the definition of the scheme $o e_{q}^{d}$ of optimistic estimates, where $d$ determines the number of classes considered at a time:

\footnotetext{
${ }^{3}$ This scheme is a generalization of the optimistic estimate proposed in GRSW08, which considers just one class at a time (instead of $d$ ).
} 


$$
\begin{gathered}
\sum_{j=1, d+1,2 d+1, \ldots}^{d}\left(\boldsymbol{p}, n, \boldsymbol{p}_{\mathbf{0}}, N\right):= \\
\max _{m_{j}^{\prime}, \ldots, m_{j+d-1}^{\prime} \mid m_{j}^{\prime} \in\left\{0, n p_{j}\right\}}\left(\operatorname { m a x } \left\{\sum_{i=j}^{j+d-1} \phi_{i}\left(\boldsymbol{m}_{-}^{\prime}, \boldsymbol{p}_{\mathbf{0}}, N\right),\right.\right. \\
\left.\left.\left.\sum_{i=j}^{j+d-1} \phi_{i}\left(\boldsymbol{m}_{+}^{\prime}, \boldsymbol{p}_{\mathbf{0}}, N\right)\right\}\right)\right]
\end{gathered}
$$

Here, the $\phi_{i}$ are summands of the quality functions as discussed in the previous section. The $\boldsymbol{m}_{-}^{\prime}$ stands for the vector $\left(0,0, \ldots, m_{j}^{\prime}, \ldots, m_{j+d-1}^{\prime}, 0, \ldots, 0\right)^{T}$ and $\boldsymbol{m}_{+}^{\prime}$ for the vector $\left(n p_{0}, n p_{1}, \ldots, n p_{j-1}, m_{j}^{\prime}, \ldots, m_{j+d-1}^{\prime}, n p_{j+d}, \ldots, n p_{c}\right)^{T}$; intuitively $\boldsymbol{m}_{-}^{\prime}$ stands for the case where the subset of $s$ includes none of the rows of the classes $1, \ldots, j-1, j+d, \ldots, c$ of the subgroup $s$, while $\boldsymbol{m}_{+}^{\prime}$ stands for the case where every row of these classes is present. Of course, if $c$ is not a multiple of $d$, the last summands might involve less than $d$ classes.

Proof. Similar to the proof for $o e_{q}^{*}$, the proof is based on the fact that every quality function considered is convex, as shown in the Appendix. Additionally, we use the fact all quality functions considered can be brought to the form $\phi_{1}\left(\boldsymbol{m}, \boldsymbol{p}_{\mathbf{0}}, N\right)+\ldots+\phi_{c}\left(\boldsymbol{m}, \boldsymbol{p}_{\mathbf{0}}, N\right)$, with $\phi_{i}$ being $\left(\sum_{j} m_{j}\right)\left(\frac{m_{i}}{\sum_{j} m_{j}}-p_{0 i}\right)^{2}$ for Split, $\frac{\left(\sum_{j} m_{j}\right)}{N-\left(\sum_{j} m_{j}\right)}\left(\frac{m_{i}}{\sum_{j} m_{j}}-p_{0 i}\right)^{2}$ for Gini and $\frac{\left(\sum_{j} m_{j}\right)}{p_{0_{i}}}\left(\frac{m_{i}}{\sum_{j} m_{j}}-p_{0 i}\right)^{2}$ for Pearson's. Now the following holds:

$$
\begin{aligned}
& \max _{m_{1}^{\prime}, \ldots, m_{c}^{\prime} \mid \forall i . m_{i}^{\prime} \in N_{+} \wedge 0 \leq m_{i}^{\prime} \leq n p_{i}}\left\{\sum_{i=1}^{c} \phi_{i}\left(\boldsymbol{m}^{\prime}, \boldsymbol{p}_{\mathbf{0}}, N\right)\right\}= \\
& \max _{m_{1}^{\prime}, \ldots, m_{c}^{\prime} \mid \forall i . m_{i}^{\prime} \in\left\{0, n p_{i}\right\}}\left\{\sum_{i=1}^{c} \phi_{i}\left(\boldsymbol{m}^{\prime}, \boldsymbol{p}_{\mathbf{0}}, N\right)\right\} \leq \\
& \sum_{j=1, d+1,2 d+1, \ldots, c} \max _{m_{1}^{\prime}, \ldots, m_{c}^{\prime} \mid \forall i . m_{i}^{\prime} \in\left\{0, n p_{i}\right\}}\left\{\sum_{i=j}^{j+d-1} \phi_{i}\left(\boldsymbol{m}^{\prime}, \boldsymbol{p}_{\mathbf{0}}, N\right)\right\} \leq \\
& \sum_{j=1, d+1, \ldots, c} \max _{m_{j}^{\prime}, \ldots, m_{j+d-1}^{\prime} \mid m_{j}^{\prime} \in\left\{0, n p_{j}\right\}}\left[\max _{m_{1}^{\prime}, \ldots m_{j-1}^{\prime}, m_{j+d}, \ldots m_{c}^{\prime} \mid m_{i}^{\prime} \in\left\{0, n p_{i}\right\}}\left\{\sum_{i=j}^{j+d-1} \ldots\right\}\right]
\end{aligned}
$$

where $\ldots$ stands for $\phi_{i}\left(\boldsymbol{m}^{\prime}, \boldsymbol{p}_{\mathbf{0}}, N\right)$. Now we make use of the fact that $\phi_{i}$ is not only convex in $m_{i}$ and $m_{j}, j \neq i$, but also in the sum of $m_{j}$ 's with $j \neq i$. That is, for every set of indexes $J=\left\{j_{1}, \ldots j_{n}\right\}$ not including $i, \phi_{i}$ is convex in $\sum_{k \in J} m_{k}$. This follows from the fact that in all definitions of $\phi_{i}$ (i.e. for the definition for Split, Gini and $\chi^{2}$ ) the $m_{j}$ with $j \neq i$ only occur in the sum $\sum_{j} m_{j}$. Thus, for any set of indexes $J$ that does not include $i, \phi_{i}$ could be considered as a function of the new parameter $\left(\sum_{k \in J} m_{k}\right)$ and the remaining $m_{k^{\prime}}$, i.e. those with index $k^{\prime} \notin J$. The resulting function is of the same form as $\phi_{i}$ (except that it takes less parameters) and thus is convex in $\sum_{k \in J} m_{k}$. 
In particular, any $\phi_{i}$ with $j \leq i \leq j+d-1$ is convex in $\sum_{k \in\{0, \ldots, j-1, j+d, \ldots c\}} m_{k}$. Therefore, it is sufficient to consider the case where the sum is minimal or maximal, that is when either all these $m_{k}^{\prime}$ 's are zero or all have value $n p_{k}$. So the above is bounded by

$\sum_{j=1, d+1, \ldots, c}\left[\max _{m_{j}^{\prime}, \ldots, m_{j+d-1}^{\prime} \mid m_{j}^{\prime} \in\left\{0, n p_{j}\right\}}\left(\max _{\left(\forall k . m_{k}^{\prime}=0\right),\left(\forall k . m_{k}^{\prime}=n p_{k}\right)} \sum_{i=j}^{j+d-1} \phi_{i}\left(\boldsymbol{m}^{\prime}, \boldsymbol{p}_{\mathbf{0}}, N\right)\right)\right]$

This is equivalent to $o e_{q}^{d}$ and the proof is completed.

Some Considerations on the new Optimistic Estimates. We will now consider some properties of the optimistic estimates $o e_{q}^{d}$. As before, we use $c$ to denote the number of classes. The computation of $o e_{q}^{d}$ involves the evaluation of $O\left(\frac{c}{d} 2^{d} d\right)=$ $O\left(c 2^{d}\right)$ different expressions $\phi_{i}$, meaning that the higher the number $d$ of classes considered at a time, the higher the computational cost. We remark that in an implementation of the function $o e_{q}^{d}$, only those classes where $m_{i}>0$ have to be considered, meaning that $O\left(c 2^{d}\right)$ is only the worst case.

Lemma 3. $o e_{q}^{2 d}$ is at least as conservative as $o e_{q}^{d}$. oe $e_{q}^{d}$ is tight if either $d \geq c$, or $c=2$ and $d \geq 1$.

Proof. It is easy to see that if $c \leq d$, then $o e_{q}^{d}=o e_{q}^{*}$, because the number of classes $d$ in $o e_{q}^{d}$ for which every combination is considered is exactly $c$, as in $o e_{q}^{*}$. $o e_{q}^{2 d}$ is at least as conservative as $o e_{q}^{d}$ because $o e_{q}^{2 d}$ considers every combination of classes considered by $o e_{q}^{d}$ (and some more combinations).

In the special case $c=2, o e_{q}^{1}$ is tight because the set of indexes $\{0, \ldots, j-1, j+$ $d, \ldots c\}$ considered in the second sum of Equation 3 actually involves only one class index, and hence effectively every combination of the two classes is considered, just as in $o e_{q}^{*}$. To show that $o e_{q}^{d}$ is not tight otherwise, it is sufficient to have one example. The experiments in Section 4 have plenty of them, and the next paragraph also presents one. Here is another one: consider $\boldsymbol{p}_{\mathbf{0}}=(0.1,0.45,0.45)$ (for $c=3$ ) respectively $\boldsymbol{p}_{\mathbf{0}}=\left(0.1,0.3, \frac{0.3}{c-3}, \ldots, \frac{0.3}{c-3}, 0.3\right)$ (for $c>3$ ). Furthermore, consider a subgroup $s$ with $\boldsymbol{m}=(10,10,0,0, \ldots 0,10)$. It is easy to verify that of all subsets of $\boldsymbol{m}, \boldsymbol{m}^{\prime}=(10,0,0, \ldots 0,0)$ has the highest quality. However, the last summand of $o e_{q}^{d}$ would consider the values 10 and 0 for $m_{c}^{\prime}$ only in combination with either both $m_{1}^{\prime}=10$ and $m_{2}^{\prime}=10$ or both $m_{1}^{\prime}=0$ and $m_{2}^{\prime}=0$. That is, it would not consider the actual maximizing combination $(10,0,0, \ldots 0,0)$, but instead will provide an overoptimistic estimate.

An Example. To illustrate the effect of different optimistic estimates, let us reconsider the example from Figure 1. In particular, let us consider the subgroup description $\{$ Region $=U$ rban $\}$. The corresponding subgroup consists of the first three rows of the dataset and has a probability distribution of $p_{H i g h}=1 / 3$, $p_{\text {Med }}=2 / 3$, and $p_{\text {Low }}=0$.

For the Split quality function, we get a tight optimistic estimate of 0.176 for the quality of the refinements of $\{$ Region $=U$ rban $\}$. Using the suboptimal 
estimate $o e_{q}^{1}$, we only get the estimate 0.255 . While both estimates can be used by a subgroup discovery algorithm, if the minimal required quality is 0.195 only the tight estimate of 0.176 would allow to prune all subgroups description below $\{$ Region $=U$ rban $\}$ (In fact, 0.195 is exactly the minimal required quality at that point, if the algorithm sketched in Section 4 is used).

\section{Experimental Evaluation}

Sketch of a Subgroup Discovery Algorithm. To evaluate the impact of different optimistic estimates, we used a branch and bound depth-first-search (DFS) algorithm similar to OPUS ${ }^{O}$ Web95 (without optimistic reordering). The optimistic estimates are used to prune as large a part of the search space as possible, and furthermore determine the order in which the nodes are expanded during DFS. Our implementation makes use of FP-Trees HPY00] to speedup the calculation of the parameters $\boldsymbol{p}$ and $n$ of a subgroup, as first proposed in AP06. The overall algorithm, called DpSubgroup, is described in more detail in GRSW08.

Datasets and Results. We evaluated the impact of the different optimistic estimates on five datasets: four datasets from the UCI Machine Learning Repository

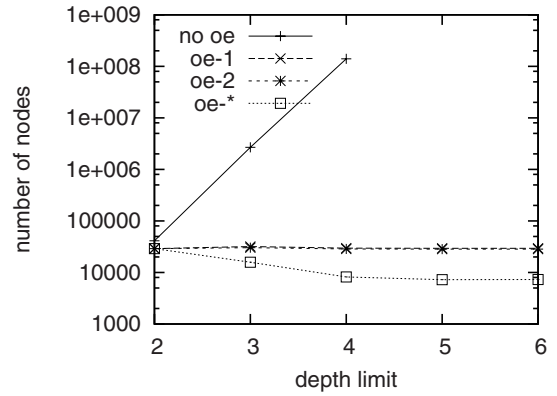

(a) \# nodes considered (Splice/Split)

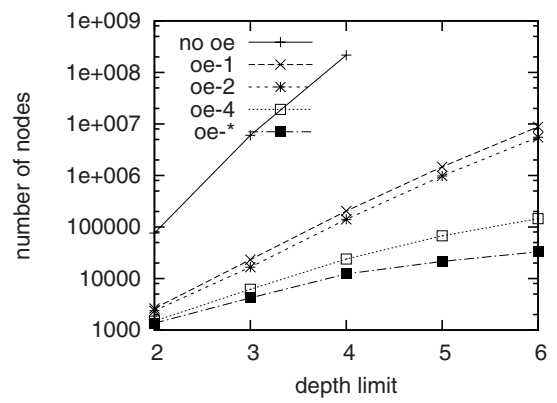

(c) \# nodes considered (Census/Gini)

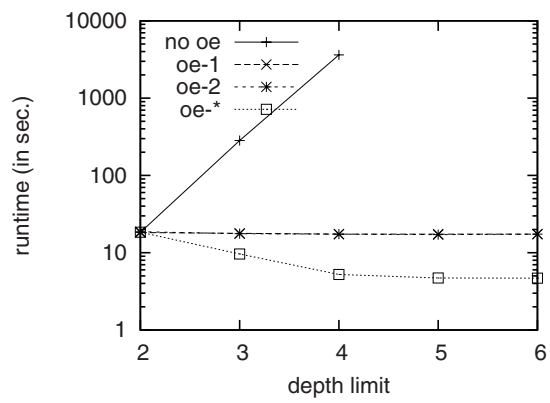

(b) Runtime (Splice/Split)

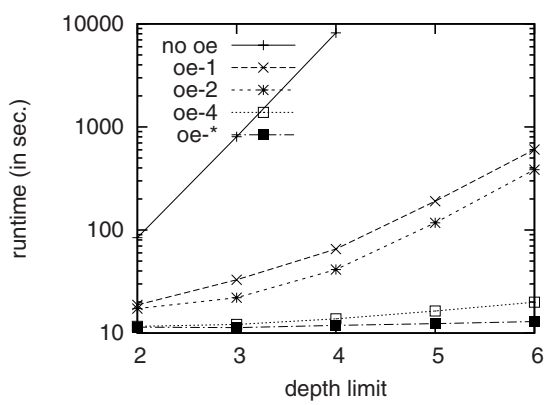

(d) Runtime (Census/Gini)

Fig. 2. Results for the Splice and Census dataset (with 3 resp. 5 classes) using the Split resp. the Gini quality function. The curves show the number of nodes considered (left images) resp. the runtime (right images) for different optimistic estimates. 
AN07 and one real-world dataset. In particular, we used the "Mushroom" dataset with 8124 rows, 22 attributes and 2 classes; the "Soybean" dataset with 683 rows, 35 attributes and 19 classes; the "splice" dataset with 3190 rows, 62 attributes and 3 classes; a sample of 30.000 rows of the UsCensus1990 database with 68 attributes and 5 classes (we used "dIncome1" as class attribute); and finally a prescription dataset with 29 attributes, 60488 rows and 11 classes from the $i$ WebCare project (http://iwebcare.iisa-innov.com/). In all experiments, we searched for the top 100 groups on an Intel Core 2 Duo T7500 with 2 GB of RAM under Windows XP.

Figure 2 shows the performance results on the datasets Census and Splice, using different quality functions. The horizontal axis shows the depth limits for the subgroup discovery, that is a maximum length of the subgroup descriptions considered. The different curves show the results using different optimistic estimates: "oe-d" stands for subgroup discovery with $o e_{q}^{d}$, "oe-*" shows the results using the tight optimistic estimate $o e_{q}^{*}$, and finally "no oe" shows the performance without any optimistic estimate pruning. It is worthwhile to note that "no oe" essentially corresponds to the algorithm SD-Map (which also makes use of FP-Trees but does not use optimistic estimate pruning), because this algorithms has been shown to outperform all other exhaustive algorithms like Apriori-SD [KLJ03] by an order of magnitude AP06.

The figure shows both the number of nodes explored during the subgroup discovery and the overall runtime, using a logarithmic scale. As expected, the number of nodes considered depends on the optimistic estimate used: The higher the degree $d$ in $o e_{q}^{d}$, the less nodes are considered. The use of the tight optimistic estimate $o e_{q}^{*}$ results in a minimal number of considered nodes 4 Similar to the number of nodes, the runtime is affected by the optimistic estimate used. Although the performance ratio does not exactly correspond to the node ratio, the ordering of the optimistic estimates is the same. The performance gain using pruning can become as large as an order of magnitude and more.

Figure 3 shows the results for the Soybean and the Mushroom dataset. We first consider the results for the Soybean dataset (subfigures (a)-(c)): Again, the higher the degree $d$ of the optimistic estimate $o e_{q}^{d}$, the less nodes are considered, with $o e_{q}^{*}$ being optimal (Figure $3(\mathrm{a})$. Regarding the runtime, however, the situation is different: Figure 3(b) shows that the runtime is minimal when a pruning level $d$ is between 1 and 4 . The reason is that although the more conservative optimistic estimates reduce the number of nodes considered, their calculation is more expensive. This effect becomes apparent in the Soybean dataset because it has much more classes than the earlier datasets.

The above experiment shows that for datasets with a large number of classes, it can be appropriate to use a non-tight optimistic estimate. However, the best pruning level depends on the ratio of the costs for the calculation of the optimistic

\footnotetext{
${ }^{4}$ It is interesting to note that the number of nodes and the runtime sometimes decreases when the depth limit increases. The reason is that the algorithm quickly finds subgroups with a very high quality at a higher level, which allows to prune a larger part of the search space than possible if only shorter subgroups were considered.
} 


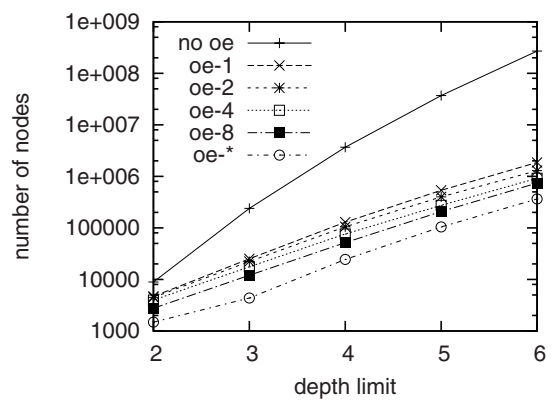

(a) \# nodes considered (Soybean/Gini)

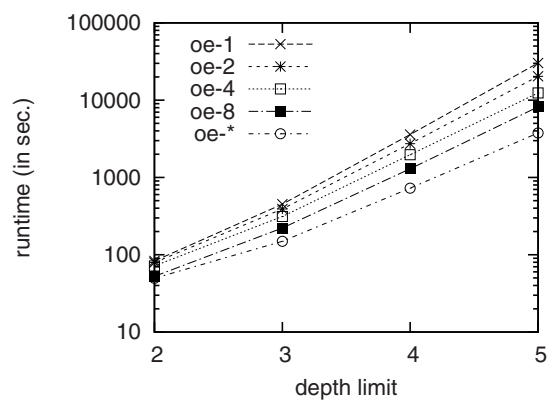

(c) Runtime using persistent FpTree

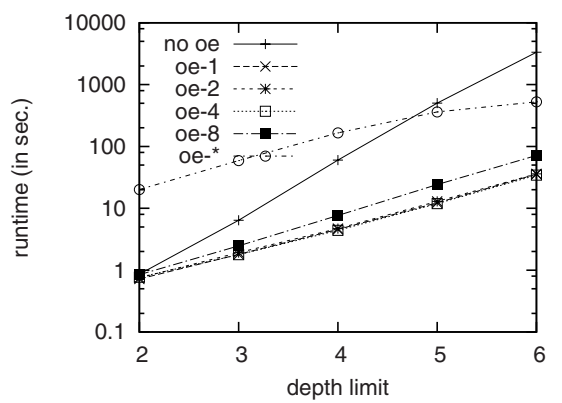

(b) Runtime using FpTree (Soybean/Gini)

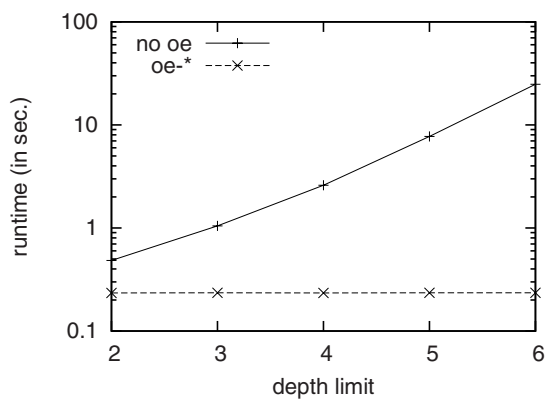

(d) Runtime (Mushroom/P.-S.)

Fig. 3. Number of nodes (a) and runtime (b, c) on the Soybean dataset (using the Gini quality function), and runtime on the mushroom dataset (d) (using the PiatetstkyShapiro quality function)

estimate, and the cost for the calculation of the parameters of a subgroup. So far we used FP-Trees, which allowed a very fast calculation of $n$ and $\boldsymbol{p}$. However, if the dataset is very large it might not be possible to keep the FP-Tree in main memory. To see how the situation changes if the calculation of the parameters $n$ and $p$ becomes more expensive, we have run some experiments using a persistent FP-Tree, i.e. an implementation where the FP-Trees are stored on disk HPYM04. Our prototypical implementation is based on the object database db4o PEHH06]. Figure 3(c) shows the resulting runtime: In this setting, the use of conservative optimistic estimates pays off again, with $o e_{q}^{*}$ resulting in the fastest calculation (the number of nodes is, of course, unaffected).

Finally, Figure 3(d) shows the runtime for the Mushroom dataset, using the Piatetstky-Shapiro quality. As in the multi-class case, the use of optimistic estimates results in a significant speedup in this two-class example. The results from the prescriptions dataset do not significantly differ, so we merely used them in the summarizing table in the next paragraph.

Summary. The above results show that the optimistic estimates presented in this paper have a significant impact on the performance of the subgroup discovery. The use of the (fastest to calculate) optimistic estimates $o e_{q}^{1}$ and $o e_{p s}^{*}$ never 
slows down the execution but instead results in a tremendous speedup. The performance gain reaches an order of magnitude at relative small depth bounds (about 2 to 4 ) and gets even larger when the depth limit continues to increase.

The use of more conservative optimistic estimates allow to further speedup the subgroup discovery. The following table summarizes the performance gain over $o e_{q}^{1}$ achieved using the optimistic estimates $o e_{q}^{d}$ and $o e_{q}^{*}$ (with $d>1$ ). For different values of $d$ (i.e. $2,3,4$ and $\infty$ ), it compared the performance with that achieved using $o e_{q}^{1}$, aggregated over different quality functions and datasets. In particular, it shows the relative runtime (the runtime using the more conservative optimistic estimate, divided by the runtime using $o e_{q}^{1}$ ) in the best experiment (Minimal), the worst experiment (Maximal) and on average. The table is based on a total of 248 experiments.

\begin{tabular}{l|c|c|c|c} 
& $o e_{q}^{2}$ & $o e_{q}^{3}$ & $o e_{q}^{4}$ & $o e_{q}^{*}$ \\
\hline Minimal relative runtime compared to $o e_{q}^{1}$ & $62 \%$ & $21 \%$ & $3 \%$ & $1 \%$ \\
Average relative runtime compared to $o e_{q}^{1}$ & $93 \%$ & $69 \%$ & $63 \%$ & $847 \%$ \\
Maximal relative runtime compared to $o e_{q}^{1}$ & $113 \%$ & $118 \%$ & $135 \%$ & $3640 \%$
\end{tabular}

The table shows that the larger $d$, the more the runtime can decrease (due to the stronger pruning) - but it can also increase (due to the computation time of the optimistic estimate). Overall, the use of $o e_{q}^{4}$ (which is tight if the number of classes is $\leq 4$ ) is a safe choice in most situations. While in the worst example, it was slower by $35 \%$ than $o e_{q}^{1}$, on average it was faster, taking only $63 \%$ of $o e_{q}^{1}$ 's runtime; In the best example, it even reduced the runtime to $3 \%$ of that of $o e_{q}^{1}$.

\section{Summary and Discussion}

In this paper, we have pursued the investigation of optimistic estimates for fast subgroup discovery, started in Wro97. In particular, we considered and formalized the concept of tight optimistic estimates. We have shown that the optimistic estimate proposed in Wro97 is not tight, and have presented new tight optimistic estimates, including tight optimistic estimates for several multi-class quality functions.

While the use of tight optimistic estimates minimizes the number of subgroups considered, their calculation sometimes becomes quite time consuming. To cope with this difficulty, we have presented a family of increasingly efficient approximations of the tight optimistic estimates. While these estimates are (in general) not tight, they allow to trade more conservative estimates for faster computation and thereby provide a mean to select an optimistic estimate with the right ratio of conservative-ness and computational cost.

The results are interesting both from a theoretic and a practical point of view. On the theoretical side, the notions of conservative and tight optimistic estimates allow to compare optimistic estimates. On the practical side, our experiments show that the use of the new optimistic estimates $o e_{p s}^{*}, o e_{q}^{d}$ and $o e_{q}^{*}$ result in a significant speedup compared to current state-of-the-art algorithms like SD-Map AP06. While for problems with a relatively small number of classes $o e_{q}^{*}$ is the estimate of choice, for datasets with a larger number of classes (more than 6 or 
so) the optimal choice depends on more factors. Overall, the use of $o e_{q}^{4}$ is a safe choice in most situations.

The idea to perform pruning based on an optimistic evaluation of the search space below a node was already investigated before the introduction of optimistic estimate into subgroup discovery. In particular, Webb Web95] considers optimistic pruning for rule learning tasks in his OPUS search algorithm. The concept is also applied in other pattern-mining tasks involving non-monotonic objective functions, like tiling databases GGM04. Of course, the objective function considered in tasks like tiling are different than in subgroup discovery.

In future work, we plan to investigate optimistic estimates for other quality function, and to extend the concept of tight optimistic estimates to numeric target attributes. We would also like to investigate whether the optimistic estimates $o e_{q}^{d}$ can be improved by some kind of heuristic grouping of the classes, with the idea not just to build arbitrary groups of $d$ attributes. It would also be interesting to combine the optimistic estimates presented in this paper with other approaches to subgroup discovery, in particular with sampling-based approaches SW00]. Altogether, we believe that the definition of tight optimistic estimates and the new optimistic estimates presented in this paper can be a valuable instrument in a wide range of subgroup discovery algorithms.

Acknowledgments. The financial support of the European Commission under the Project $i W e b C a r e$ (IST-2005-028055) is gratefully acknowledged.

\section{References}

[ABP06] Atzmüller, M., Baumeister, J., Puppe, F.: Introspective subgroup analysis for interactive knowledge refinement. In: Sutcliffe, G., Goebel, R. (eds.) FLAIRS Conference, pp. 402-407. AAAI Press, Menlo Park (2006)

[AN07] Asuncion, A., Newman, D.J.: UCI machine learning repository (2007)

[AP06] Atzmüller, M., Puppe, F.: SD-map - a fast algorithm for exhaustive subgroup discovery. In: Fürnkranz, J., Scheffer, T., Spiliopoulou, M. (eds.) PKDD 2006. LNCS (LNAI), vol. 4213, pp. 6-17. Springer, Heidelberg (2006)

[BFOS84] Breiman, L., Friedman, J.H., Olshen, R.A., Stone, C.J. (eds.): Classification and Regression Trees. Wadsworth (1984)

[Bre96] Breiman, L.: Technical note: Some properties of splitting criteria. Machine Learning 24(1), 41-47 (1996)

[BV04] Boyd, S., Vandenberghe, L.: Convex Optimization. Cambridge University Press, Cambridge (2004)

[GGM04] Geerts, F., Goethals, B., Mielikäinen, T.: Tiling databases. In: Suzuki, E., Arikawa, S. (eds.) DS 2004. LNCS (LNAI), vol. 3245, pp. 278-289. Springer, Heidelberg (2004)

[GRSW08] Grosskreutz, H., Rüping, S., Shaabani, N., Wrobel, S.: Optimistic estimate pruning strategies for fast exhaustive subgroup discovery. Technical report, Fraunhofer Institute IAIS (2008), http://publica. fraunhofer.de/eprints/urn:nbn:de:0011-n-723406.pdf 
[HPY00] Han, J., Pei, J., Yin, Y.: Mining frequent patterns without candidate generation. In: Chen, W., Naughton, J.F., Bernstein, P.A. (eds.) SIGMOD Conference, pp. 1-12. ACM, New York (2000)

[HPYM04] Han, J., Pei, J., Yin, Y., Mao, R.: Mining frequent patterns without candidate generation: A frequent-pattern tree approach. Data Min. Knowl. Discov. 8(1), 53-87 (2004)

[KLGK07] Kralj, P., Lavrac, N., Gamberger, D., Krstacic, A.: Contrast set mining through subgroup discovery applied to brain ischaemina data. In: Zhou, Z.-H., Li, H., Yang, Q. (eds.) PAKDD 2007. LNCS (LNAI), vol. 4426, pp. 579-586. Springer, Heidelberg (2007)

[KLJ03] Kavsek, B., Lavrac, N., Jovanoski, V.: Apriori-sd: Adapting association rule learning to subgroup discovery. In: R. Berthold, M., Lenz, H.-J., Bradley, E., Kruse, R., Borgelt, C. (eds.) IDA 2003. LNCS, vol. 2810, pp. 230-241. Springer, Heidelberg (2003)

[Klö96] Klösgen, W.: Explora: A multipattern and multistrategy discovery assistant. In: Advances in Knowledge Discovery and Data Mining, pp. 249-271 (1996)

[Klö02] Klösgen, W.: Subgroup Discovery. In: Handbook of Data Mining and Knowledge. Oxford University Press, Oxford (2002)

[KM02] Klösgen, W., May, M.: Spatial subgroup mining integrated in an objectrelational spatial database. In: Elomaa, T., Mannila, H., Toivonen, H. (eds.) PKDD 2002. LNCS (LNAI), vol. 2431, pp. 275-286. Springer, Heidelberg (2002)

[LCGF04] Lavrac, N., Cestnik, B., Gamberger, D., Flach, P.A.: Decision support through subgroup discovery: Three case studies and the lessons learned. Machine Learning 57(1-2), 115-143 (2004)

[LKFT04] Lavrac, N., Kavsek, B., Flach, P., Todorovski, L.: Subgroup discovery with cn2-sd. Journal of Machine Learning Research 5, 153-188 (2004)

[PEHH06] Paterson, J., Edlich, S., Hörning, H., Hörning, R.: The Definitive Guide to db4o. Apress, Berkely (2006)

[SW00] Scheffer, T., Wrobel, S.: A sequential sampling algorithm for a general class of utility criteria, pp. 330-334. ACM Press, New York (2000)

[Web95] Webb, G.I.: Opus: An efficient admissible algorithm for unordered search. J. Artif. Intell. Res (JAIR) 3, 431-465 (1995)

[Wro97] Wrobel, S.: An algorithm for multi-relational discovery of subgroups. In: Komorowski, J., Żytkow, J.M. (eds.) PKDD 1997. LNCS, vol. 1263, pp. 78-87. Springer, Heidelberg (1997)

\section{A Proof of Convexity of the Multi-class Quality Functions}

\section{A.1 Split and Pearson's $\chi^{2}$}

Both the Split and Pearson's $\chi^{2}$ quality functions are nonnegative weighted sums. The nonnegative weighted sum of convex functions is convex ([BV04]), hence it is sufficient to show that the summands are convex. The summand of both quality functions can be brought to the following form

$$
\phi_{i}=c_{i}\left(\sum_{j} n_{j}\right)\left(\frac{n_{i}}{\sum_{j} n_{j}}-p_{0 i}\right)^{2}
$$

where in the case if Split $c_{i}=1$ and in the case of Pearson's $c_{i}=\frac{1}{p_{0_{i}}}$. 
We only need to consider the case where $c_{i}=1$, because the $c_{i}$ 's are merely positive weights. The $\phi_{i}$ 's are twice differentiable, thus it is sufficient to show that the Hessian or second derivative is positive semidefinite BV04. We only consider the first summand $\phi_{1}$, as the other cases are analog. It is somewhat laborious but straightforward to verify that

$$
\nabla^{2} \phi_{1}=\frac{2}{\left(\sum_{j} n_{j}\right)^{3}}\left[\begin{array}{cccc}
\left(\sum_{j \neq 1} n_{j}\right)^{2} & -n_{1}\left(\sum_{j \neq 1} n_{j}\right) & \ldots & -n_{1}\left(\sum_{j \neq 1} n_{j}\right) \\
-n_{1}\left(\sum_{j \neq 1} n_{j}\right) & \left(n_{1}^{2}\right) & \ldots & \left(n_{1}^{2}\right) \\
\ldots & \ldots & \ldots & \ldots \\
-n_{1}\left(\sum_{j \neq 1} n_{j}\right) & \left(n_{1}^{2}\right) & \ldots & \left(n_{1}^{2}\right)
\end{array}\right]
$$

The above matrix can be brought to the following form

$$
G G^{T}
$$

with $G=\left(\sum_{j \neq 1} n_{j},-n_{1}, \ldots,-n_{1}\right)^{T}$, that is there is a Cholesky decomposition and hence the matrix is positive-definite BV04.

\section{A.2 Gini}

The fact that the Gini quality function is convex can be derived from previous work in the area of decision tree construction. This comes from the fact that the Gini quality functions is based on the Gini index, used as a splitting criterion in the construction of decision trees Bre96, BFOS84. The Gini index is defined as $G(p)=\sum_{j} p_{j}\left(1-p_{j}\right)$ and measures the "impurity" of a distribution $p$. The gain in impurity resulting from a split is defined as $\Theta(s)=G\left(\boldsymbol{p}_{\mathbf{0}}\right)-P_{L} G\left(\boldsymbol{p}_{\boldsymbol{l}}\right)-$ $P_{R} G\left(\boldsymbol{p}_{\boldsymbol{r}}\right)$ and was used as a goodness of a split measure. Here, $\boldsymbol{p}_{\mathbf{0}}$ denotes the distribution over the classes in the overall population, $\boldsymbol{p}_{\boldsymbol{l}}$ and $\boldsymbol{p}_{\boldsymbol{r}}$ denote the distribution in the left and the right subpopulation resulting from a split, $P_{L}$ denotes the proportion of the population send to the left by the split and $P_{R}=$ $1-P_{L}$ denotes the proportion send to the right.

This goodness of split was turned into the Gini quality function Klö96:

$$
G\left(\boldsymbol{p}_{\mathbf{0}}\right)-g * G(\boldsymbol{p})-(1-g) * G\left(\boldsymbol{p}^{*}\right)
$$

where $\boldsymbol{p}_{\mathbf{0}}$ is (as before) the class distribution in the overall population, $\boldsymbol{p}$ the class distribution in the subgroup, $\boldsymbol{p}^{*}$ the probability distribution in the remainder, i.e. in the examples from the overall population not in the subgroup, and finally $g$ the generality of the subgroup. It is easy to verify that the components of $p^{*}$ are defined by $p_{i}^{*}=\frac{N p_{0_{i}}-N g p_{i}}{N(1-g)}=\frac{p_{0_{i}-g p_{i}}}{1-g}$. By inserting this definition of $p^{*}$ we obtain the following, more familiar-looking definition of the Gini quality,

$$
\frac{g}{1-g} \sum_{j}\left(p_{i}-p_{0 i}\right)^{2}=\frac{n}{N-n} \sum_{j}\left(p_{i}-p_{0 i}\right)^{2}
$$

Bre96 shows that $g * G(\boldsymbol{p})+(1-g) * G\left(\boldsymbol{p}^{*}\right)$ is concave in the proportion of the probabilities that are sent to the left (which he calls $\alpha$ ). We remark that Breiman 
uses the term convex in the meaning of "convex downward"; We use the term convex in the opposite sense, as BV04 (Breimans notation also differs from ours in other respects). Now our vector $\boldsymbol{m}$ can be obtained from $\alpha$ by an affine mapping, namely $m_{i}=N p_{0 i} \alpha_{i}$, which implies that $g * G(\boldsymbol{p})+(1-g) * G\left(\boldsymbol{p}^{*}\right)$ is also concave in $\boldsymbol{m}$, because affine mappings preserves concavity. Hence the Gini quality function is convex in $\boldsymbol{m}$. 